carefully check whether or not animals (or their parts) declared as captive-bred have indeed been bred under controlled conditions.

VINCENT NIJMAN School of Social Sciences and Law, Department of Anthropology and Geography, Oxford Brookes University, Oxford, UK

E-mailvnijman@brookes.ac.uk

\section{Photographic database informs management of conflict tigers}

The increasing interface between people and wildlife contributes to human-wildlife conflict in many conservation landscapes. In India animals suspected to be involved in conflict are often captured and translocated to different locations or zoological parks. A key concern in the capture of so-called problem animals has been the identification of individuals involved in conflict. The Wildlife Conservation Society (WCS) India Program, in collaboration with research partners, has aided government officials in this matter through its research on tigers in the Malenad Tiger Landscape, south-west India. As part of a research programme initiated in 1991, WCS India has formulated a protocol for individual identification of tigers, based on their stripe patterns. Using pattern-matching software that aids quick and reliable identification of tigers from photographs (Hiby et al., 2009, Biology Letters, 5, 383-386), a photographic database has been developed, with $>750$ individually identified tigers from 16 protected areas totalling $>38,000 \mathrm{~km}^{2}$, and elsewhere, in India. Ancillary information on home ranges, activity patterns and sociobiology of conflict-tigers provides a unique opportunity for informed management of the species. This long-term, ongoing study is funded by the Department of Science and Technology and the Department of Biotechnology of the Government of India, Vision Group on Science \& Technology of the Government of Karnataka, and the Wildlife Conservation Society, New York.

Two recent cases of human-tiger conflict in the Malenad Tiger Landscape exemplify the utility of this database and long-term monitoring for conflict mitigation. One incident, on 27 December 2013, involved the loss of human life in Bandipur National Park, and the other, on 1-2 January 2014, involved the killing of cattle in a village adjoining Nagarahole National Park. In the latter incident a dead cow was used as bait to capture the tiger involved. In the incident in Bandipur photographs from camera traps set up in the area of conflict were matched to individuals in the database. One individual was identified in nine of the 15 photographs obtained and, as the location of conflict was well beyond its home range, it was identified as the conflict individual and was subsequently captured. The tigers from both incidents have been transferred to a nearby zoo. The Forest Department debated relocation of the individuals but this was not pursued.

Both of the tigers involved in these incidents had been photographed previously multiple times. The tiger in Bandipur had been photographed over the last 5 years and was probably an old individual past its prime. The tiger in Nagarahole was 2-3 years old and had only been photographed in the previous year. The distances between the locations of previous photographs and the location of conflict were $4-8 \mathrm{~km}$ for the tiger in Bandipur and $35 \mathrm{~km}$ for the tiger in Nagarahole. The observation of the Nagarahole individual with another male in two photographs suggests that this individual was dispersing from its natal home range to establish a breeding territory.

Nagarahole and Bandipur National Parks have high tiger densities (10-15 tigers per $100 \mathrm{~km}^{2}$ ) with c. $20 \%$ of the population lost annually to mortality and emigration. It is likely that the Nagarahole individual was emigrating from the reserve. The infrequency of dispersal events, particularly in human-dominated landscapes such as the Malenad Tiger Landscape, suggests that the removal of the Nagarahole tiger, a potential long-distance disperser, may have detrimental effects on the long-term population viability of tigers in this landscape. In contrast, the removal of the Bandipur tiger will probably have negligible effects on the population, although any relocation into an existing tiger population would be likely to cause further conflict (Athreya et al., 2011, Conservation Biology, 25, 133-141). Mitigation of conflict is frequently ad hoc, with management authorities pressurized into quick but often ineffective action. In these two contrasting examples, alternative actions could have had very different consequences. In areas where data on the age, reproductive status and ranging patterns of conflict animals are available, this information should be used to direct conflict mitigation strategies such that they are maximally effective whilst inflicting least harm on species viability.

K. Ullas Karanth, N. Samba Kumar and Divya VasudeV Wildlife Conservation Society, India Program, Bangalore, India. E-mail vasudev.divya@gmail.com

\section{First village successfully resettled from Dandeli-Anshi Tiger Reserve}

Much debate on the conservation of species such as the tiger has centred around the need for inviolate spaces devoid of human presence. A conservation strategy that emerges from this is the resettlement of villages from within the core zones of tiger conservation areas. The resettlement of such villages not only frees up space for dedicated tiger conservation but provides the resettled people with better access to amenities and serves to mitigate human-wildlife conflict (Karanth, 2007, Biological Conservation, 139, 315-324). Although 